\title{
Performance Monitoring of Service-Level Agreements for Utility Computing Using the Event Calculus
}

\author{
Andrew D H Farrell, Marek J Sergot, \\ Department of Computing, \\ Imperial College London, \\ United Kingdom. \\ \{adf02, mjs\}@doc.ic.ac.uk
}

\author{
Mathias Salle, Claudio Bartolini, \\ HP Labs, \\ Palo Alto, California, USA. \\ \{mathias.salle, claudio.bartolini\}@ @p.com
}

\begin{abstract}
Utility Computing (UC) is concerned with the provisioning of computational resources (compute-power, storage, network bandwidth), on a per-need basis, to corporate businesses. Service-level Agreements (SLAs) contracts between a provider and a customer - are a sine qua non in the deployment of UC. A crucial stage in the life-cycle of contracts (such as SLAs) is their automated performance monitoring while active; a significant aspect of which concerns the tracking of contract state.

In this work, we define an ontology to capture aspects of SLAs that are pertinent to the tracking of state for performance monitoring, and generalise these aspects so that the ontology may be applicable to other contract domains. The ontology is formalised as an XML-based language, called CTXML (contract tracking XML). The semantics for CTXML are presented in terms of a computational model based on the Event Calculus.
\end{abstract}

\section{Introduction}

Utility Computing (UC) [10] offers an opportunity to corporate businesses to maximise the efficiency and efficacy of their IT service provision (both in-house and to customers). It will allow them to out-source large areas of their IT service provision to UC-data centres, which will agree to provide computational resources, packaged as services to them.

The levels of service that are agreed between a UC service-provider and customer are mandated by Qualityof-Service (QoS) guarantees, written as service-level objectives within Service-Level Agreements (SLAs). SLAs are essential for formalising the objectives of a UC service, and to manage expectations [12].

The work that has been realised here has been concerned with one particular aspect of the life cycle of a contract (such as an SLA), namely, automated run-time performance monitoring [6]. In our view, performance monitoring is concerned with (at least) two functional aspects: (i) Tracking the effect of events (pertinent to a contract) on contract state - the contractual (or, normative) relations that hold between contract parties and informing interested parties of past, present and (possible) future contract states; and, (ii) Assessing the current state of the contract, in terms of its utility (that is, worth), and other metrics related to business intelligence [14]. The work presented in this paper is primarily concerned with the first of these, which is known as automated contract (state) tracking to distinguish it.

Notably, approaches to automated tracking of contract state, thus far, can be largely characterised in one of two ways [7]: (i) As general-purpose reasoning frameworks that (mainly) have not been applied in actual, deployed systems; or (ii) In the case of SLAs, as being fairly limited in capability. The work presented here is considered to be distinguished from such approaches in that: (i) It has been developed in the context of a 'real-world' deployment scenario (namely, SLAs for UC), while being generalised so to be applicable to other domains; and (ii) It represents an advance (over many approaches) in what can be realised regarding performance monitoring for contracts.

This paper is structured as follows. Firstly, (in section 2 ), the conceptualisation of contracts that has been used in this work is presented; followed (in section 3) by an example contract (namely, an SLA for a UC scenario), used to ground our discussions. Then, (in sections 4 and 5), a description of the contract tracking ontology, developed in this work, and its semantics are given. The paper proceeds to describe implementation and related work (in sections 6 and 7), and concludes (in section 8). 


\section{Contracts conceptualised}

It is a useful abstraction to consider that contracts (such as SLAs) are comprised of norms. A norm may be defined as: "a principle of right action binding upon the members of a group and serving to guide, control, or regulate proper and acceptable behaviour" [1].

In our work, we consider norms to be templates, which can be instantiated to yield (normative) relations that hold between contract parties. An example might be the norm: 'a service consumer is obliged to pay for service provision'. When instantiated, it yields a relation that now holds between a service consumer and provider that is, that the consumer is obliged to pay for service provision. In time, the norm may be instantiated again, creating a further relation. In fact, it may be that the first relation persists (i.e., the consumer is yet to fulfil their original obligation to pay), meaning that there is now more than one relation pertaining to the same norm.

In this presentation, it will be assumed that at most one relation pertaining to a norm may exist at any time. This is for convenience; the general case is treated in [7]. For simplicity, the existence of a relation pertaining to a norm will be described as the norm being active, and the lack of an extant relation will be described as the norm being inactive.

Crucially, it is considered here that: (i) a contract expresses norms between contract parties, whereby the actual state of the contract at any time is determined by which norms are active; (ii) norms within a contract will define the effects on the contract state of events that are presented to the contract (contract events).

\section{Example contract}

In this paper, we use the following Mail Service UC SLA in order to ground our discussions:

- The Service Provider (SP) will provide a mail service to the Service Consumer (SC), which includes a mailbox with a quota of $s$ GBytes. SC will be charged a fixed monthly fee of $s x c_{0}$ for the service.

- Whenever $u>s$, where $u$ is the mailbox utilisation in GBytes, SP will charge SC $c_{1}$ for each GByte over $s$, calculated daily, until $u \leq s$

- Whenever $u>s+e$, where $e$ is a level of tolerance in GBytes, SC will not be able to receive emails.

- In the case that the mail service is unavailable, SP is obliged to restore it within $t$ minutes. SP will pay $\$ p$ for every $t$ minutes that it is unavailable. SP is obliged to pay any penalties to SC within a month of their accruement.

- All billing of SC occurs monthly, and SC is given a month thereafter to pay. If SC fails to pay within the given time, SP may terminate the mailbox service without notice.

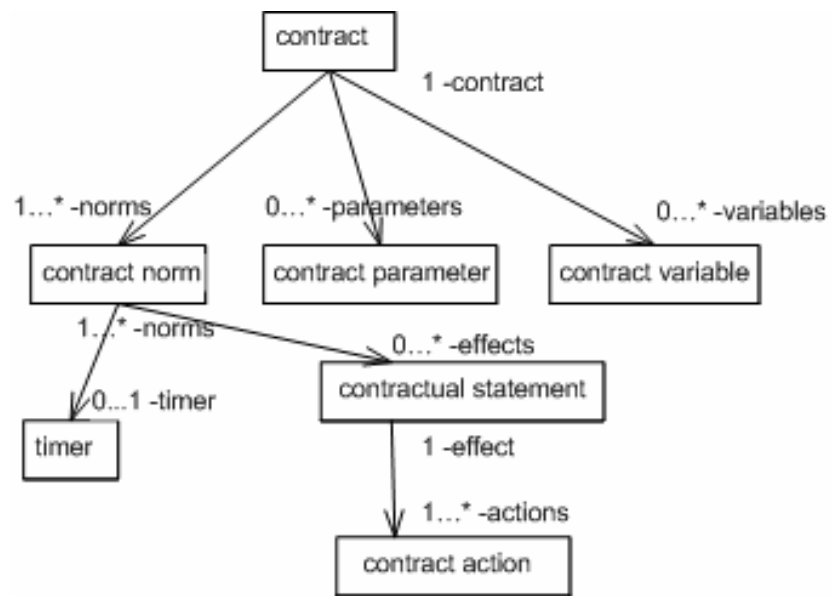

Figure 1: Contract Tracking Ontology

\section{Contract tracking ontology}

Figure 1 presents the contract tracking ontology that has been devised in this work. The ontology has also been formalised as an XML-based contract language, called CTXML (contract tracking $X M L$ ).

With reference to Figure 1, a contract is conceived as consisting of one or more contract norms, as well as zero or more contract parameters - which allow for the customisation of a contract for a particular instantiation context - and zero or more contract variables - which are used to maintain live, numerical contract state (their use is normative in that it is agreed by all parties when the contract is signed).

A contract norm may be considered as corresponding to one of many (Holfeldian-inspired) normative concepts, including (non-exhaustively): obligation, privilege, entitlement or power (see [7]). A contract norm will usually specify one or more of the following:

- One or more contractual statements, which define the effect of contract events (pertaining to the norm) on the contract. It is considered that a norm is triggered by a contract event that pertains to it.

- A timer for the norm, which is possibly recurrent.

- One or more parameters. That is, a contract norm may be parameterised. Whenever a parameterised norm is triggered by a contract event, the event will be used as the source of data for these parameters. Contractual statements contained within the norm may make use of such data.

In our work, we have considered the following conceptualisations of contract norms to be useful for the representation of contracts: 
- Contract management norms, of which we define two types: Periodic and Event

- Obligation norms

- $\quad$ Privilege norms

In turn, contract management norms (CMNs) represent the principal means of defining the effects of contract events on contract state. They contain a single contractual statement, which is executed when the norm is triggered. Note that a CMN will either be conceptualised as an event $\mathrm{CMN}$, or a periodic CMN. An event $\mathrm{CMN}$ is triggered by an external event. Contrastingly, a periodic CMN describes a (possibly recurring) timer, which triggers the norm.

An obligation norm is concerned with an obligation that bears on a party to perform one or more (noncontractual) actions. It will typically contain a contractual statement that specifies the effects on the contract in case of violation of the obligation norm, and a contractual statement that specifies the effects on the contract in case of fulfilment of the norm. It is considered that such a norm is triggered by violation and fulfilment events. An obligation norm will also specify a timer for the actions associated with the obligation to be performed by the pertinent party. Like a CMN, an obligation norm may be parameterised.

A privilege norm is concerned with (non-contractual) actions that a party is permitted to perform. It is considered illegal behaviour for a party to carry out a (non-contractual) action for which it does not have the privilege. (As a consequence, there does not exist a need for explicit prohibition norms). Furthermore, a privilege norm is considered to be a vested privilege in that other parties undertake that they will not attempt to prevent the bearer of the privilege from exercising it.

Note that, in CTXML, events - which are input to the contract - take the form:

<event id="(norm, qualification)">

$<$ para name $=" . . . ">\ldots . .</$ para $>.$.

$<$ para name $=" . . . ">\ldots</$ para $>$

$<$ /event $>$

where norm is the unique pertaining norm, and qualification is a qualification for the event - which names the contractual statement in the norm to be executed. An event may also contain associated parameters representing event data, which is passed to the contractual statements contained within any norm that the event triggers. In the sequel, the syntax for an event is abbreviated to: (norm, qualification, parameters) for simplicity, where parameters is elaborated simply as a list of parameter names.

Examples of these norms represented in CTXML for the Mail Service SLA (introduced in section 3) are now presented.
- A periodic $\mathrm{CMN}$, pcmn3, defining its (recurrent) timer as being specified by the pcnm3timer timer clause; and specifying its (single) contractual statement to be: pcmn3timeout, which is executed whenever (pcmn3, timeout, []) contract events occur. These events are generated internally according to pcnm3timer.

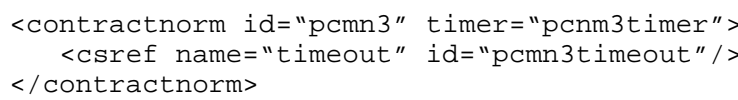

This norm in part facilitates: "SP will pay $\$ p$ for every $t$ minutes that it is unavailable" in the example SLA.

- An event $\mathrm{CMN}$, ecmn1, specifying a single contractual statement: ecmn1trigger which is executed whenever (ecmn1, trigger, [Charge]) contract events occur; and denoting that it is parameterised with a single parameter: Charge.

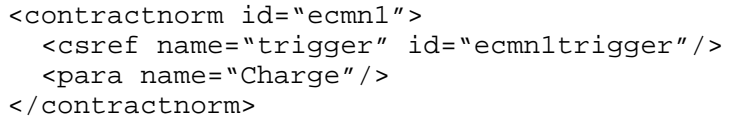

This norm in part facilitates: "SP will charge SC $\$ c_{l}$ for each GByte over $s$, calculated daily, until $u \bullet s$ ", in the example SLA.

- An obligation norm, o2, defining its (one-off) timer as being specified by the o2timer timer clause; contractual statements for non-fulfilment (violation) and fulfilment of the obligation within the time specified by o2timer as being specified by the o2violation and o2fulfilment contractual statements, respectively - executed in response to $(02$, violation, [Charge]) and (02, fulfilment, [Charge]) contract events; and denoting that it is parameterised with a single parameter: charge.

This norm in part facilitates: "SP is obliged to pay any penalties to SC within a month of their accruement" in the example SLA.

- A privilege norm, $\mathrm{p} 1$. <contractnorm id="p1"/>

This norm in part facilitates: "If SC fails to pay within the given time, SP may terminate the mailbox service without notice" in the example SLA.

A timer clause is used to specify (a recurrent, or oneoff) timer for periodic CMNs and obligation norms. Such a clause consists of one or more run clauses, which each specify a certain number of iterations of a particular timer duration. If the number of iterations is not explicitly specified (as in the example below), the run is considered to be indefinitely recurring according to the specified 
timer duration. An example of such a clause is now given, from the CTXML representation of the Mail Service SLA, for the timer used for contract norm: pcmn2. Here, the clause simply says that the timer will be indefinitely recurring with a period of 1 month.

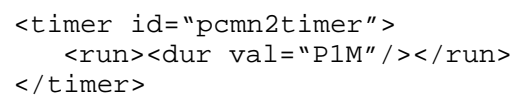

This clause in part facilitates: "All billing of SC occurs monthly..." in the example SLA.

A contractual statement clause comprises a list of contract actions, which are actions to be performed on the contract, in response to contract events. A contract action may be one of the following clauses (where the first three are considered to be atomic contract actions):

- <activate id="norm"> activation parameters $</$ activate $>$ - activates norm with given activation parameters.

- <deactivate id="norm"/> - deactivates contract norm.

- <assign id="cvar"> expr </assign> - assigns a numerical value, given by expr, to contract variable cvar.

- <ifcond then="..." else="..." /> - specifies a conditional contract action.

An example of a contractual statement, with associated contract actions, represented in CTXML for the Mail Service SLA is now presented.

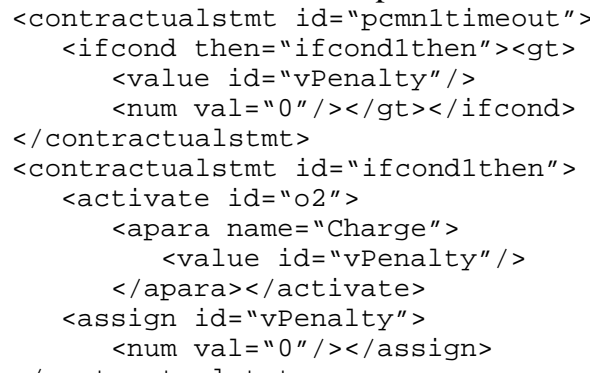

Here, the penm1timeout contractual statement consists of a single contract action - an ifcond. The ifcond action specifies a contractual statement, ifcond1then, to be performed if the condition of the ifcond holds. (It is possible for ifcond actions to also specify a contractual statement to be performed if the condition does not hold). The condition of the ifcond, in the example, stipulates contract variable vPenalty be greater than 0 . The ifcond1then contractual statement consists of a couple of contract actions - an activate action (for activating parameterised obligation norm 02 with activation parameter charge assigned to the current value of contract variable vPenalty), and an assign action (for resetting the value of the contract variable).
Finally, a contract may specify a list of initialising operations (itself a contractual statement - constrained to contain just activate operations), which are carried out on the contract when it is instantiated. Note that all contract norms are inactive, by default. As such, any norm that is required to be initially active should have a corresponding activate operation specified in this list.

\subsection{Specialisation to SLA context}

It useful to explicate an additional concept, which has been utilised within this work, that is specific to the context of representing SLAs. The concept is a servicelevel norm (SLN), which is a variation of an event CMN. An SLN encapsulates a 'service-level objective' (SLO), which defines a level of service that must be upheld throughout the lifetime of the SLA. An SLN also defines up to two contractual statements. One that specifies contract actions that are to be performed in case of violation of the (service level objective pertaining to the) SLN, and another that specifies contract actions that are to be performed in case of restoration of the SLN. An example of an SLN represented in the Mail Service SLA is now presented, where it is triggered by $(\sin 1$, violation,_) and (sln1, restoration, _) contract events.

<contractnorm id="sln1">

<csref name="violation" id="sln1viol"/>

<csref name="restoration" id="sln1rest"/> $</$ contractnorm $>$

This clause in part facilitates: "The Service Provider...a mail-storage facility of up to $s$ GBytes" and "In the case of unavailability of the mail service..." in the example SLA.

For the whole example SLA, written in CTXML, see [16].

\section{Semantics}

The semantics attributed to the contract tracking ontology are presented in terms of how the execution of contractual statements, in response to contract events, changes the state of the contract. This is achieved by describing the computational model for determining the state of norms, in the context of a narrative of contract events, according to the contractual statements contained within a contract. The computational model that is described here is inspired by the Event Calculus (EC) [11].

\subsection{Event Calculus overview}


There are many variations on the Event Calculus (EC). In the sequel, we define an XML formalisation of a simplified form of the version described in [15], called ecXML.

A contract in $e c X M L$ is a conjunction of:

- A finite set of initially clauses of the form:

<initially>

$<$ fluent id $=" F^{\prime \prime}>\ldots</$ fluent $>$

$</$ initially $>$

meaning that (boolean) fluent $F$ holds initially. (A

fluent is a property of a domain which can be attributed a value, where the value of the fluent is able

to change over time). Multi-valued fluents are assigned an initial value using similar clauses.

- A finite set of happens clauses of the form:

$<$ happens time $=" T$ " $>$

$<$ event ... $>$... $<$ /event $>$

$</$ happens $>$

meaning that the given event happened at time $T$

- A finite set of initiates clauses of the form:

<initiates>

<event ... $>$...</event $>$

$<$ fluent id=" $F ">\ldots</$ fluent $>$

condition

$</$ initiates $>$

meaning that the given event initiates fluent $F$ (makes true) if condition holds. Similar clauses can be written giving how multi-valued fluents are initiated.

- A finite set of terminates clauses of the form:

$<$ terminates $>$

$<$ event ... $>$...</event $>$

$<$ fluent id=" $F ">$ ".. $</$ fluent $>$

condition

$</$ terminates $>$

meaning that the given event terminates fluent $F$ (makes false) if condition holds. Similar clauses can be written giving how multi-valued fluents are terminated.

Additionally the following axioms (for which a full XML formalisation is neither necessary nor appropriate) are defined for ecXML:

- holds $(F, T)$ if initiated $(F, T 1, T)$ and not

terminated $(F, T 1, T)$

meaning that fluent $F$ holds at time $T$ if fluent $F$ is initiated at time $T 1$ before, or at, time $T$ and it is not terminated at a time later than $T 1$ but before, or at, time T. A similar axiom exists for multi-valued fluents. Note that it is the holds axiom, which provides the means for querying the state of a contract at any time, and thus which realises the primary purpose of applying an EC-based semantics.

- $\quad$ initiated $(F, 0, \ldots)$ if

<initially>

$<$ fluent id=" $F ">$ " < fluent $>$

$</$ initially $>$

meaning that fluent $F$ is initiated at time 0 if

fluent $F$ holds initially (as determined by any extant $e c X M L<$ initially $>$ clause for $F$ in the contract). A similar axiom exists for multi-valued fluents.

- initiated $(F, T 1, T)$ if happens $(E, T 1)$ and $T \geq T 1>0$ and

<initiates>

<event ... $>$...</event

$<$ fluent id=" $F$ " $>\ldots</$ fluent $>$

$</$ initiates $>$

meaning that fluent $F$ is initiated at time $T 1$ before, or at, time $T$, and greater than 0 , if an event $E$ happens at $T 1$ and $E$ initiates $F$ (as determined by any extant $e c X M L$ <initiates> clauses for $F$ in the contract). A similar axiom exists for multi-valued fluents.

- terminated $(F, T 1, T)$ if happens $(E, T 2)$ and $T \geq T 2>T 1$ and

$<$ terminates $>$

<event ... $>$...</event $>$

$<$ fluent id $=" F^{\prime \prime}>$... $</$ fluent $>$

$</$ terminates $>$

meaning that fluent $F$ is terminated at time $T 2$ later than $T 1$ and before, or at, time $T$ if an event $E$ happens at $T 2$ and $E$ terminates $F$ (as determined by any extant $e c X M L<$ terminates $>$ clauses for $F$ in the contract). A similar axiom exists for multi-valued fluents.

\subsection{Event Calculus based semantics}

As stated, the Event Calculus (EC) is used to provide a computational model for CTXML contractual statements. This is achieved by defining a mapping between contractual statements and expressions in EC. Note that, a contractual statement will have a distinct mapping for each contract norm to which it pertains.

Recall from section 4 that a contractual statement consists of the following types of contract actions: activate, deactivate, assign and ifcond. The mapping for the first three contract actions - the atomic actions - is now presented.

- <activate id="norm"> activation parameters

$</$ activate $>$ is mapped to:

<initiates>

<event id="(pnorm, qualification)"> parameters </event>

$<$ fluent id="norm" $>$ activation parameters

$</$ fluent $>$

$</$ initiates $>$

where (pnorm, qualification) is the event id that triggers the contractual statement with name: qualification within contract norm: pnorm; and norm is the norm activated with the given activation parameters.

- <deactivate id="norm"/> is mapped to: $<$ terminates>

<event id="(pnorm, qualification)"> parameters </event $>$

$<$ fluent id="norm" $/>$

$</$ terminates $>$ 
where norm is the norm deactivated.

- <assign id="cvar">expr</assign> is mapped to: <initiates>

<event id="(pnorm, qualification)"> parameters </event>

$<$ mvfluent id="cvar" $>$ expr $</$ mvfluent $>$ $</$ initiates $>$

where cvar is the contract variable assigned to expr.

Ifcond actions conceptually take the form: $\Delta \rightarrow \theta_{\text {then }} ; \theta_{\text {else }}$. $\Delta$ is a boolean condition on the state of norms (inactive or active) in the contract and contract events. $\theta_{\text {then }}$ is a contractual statement that is executed should the condition hold when the ifcond is executed. $\theta_{\text {else }}$ is a contractual statement that is executed if the condition fails to hold. In mapping ifcond actions to EC, $\Delta$ becomes an additional condition placed on each contract action in $\theta_{\text {then }}$; and not $\Delta$ becomes an additional condition placed on each contract action in $\theta_{\text {else }}$. Generally speaking, there may be an arbitrary nesting to an ifcond action meaning that any atomic activate, deactivate, or assign actions specified within may be subject to a number of boolean conditions: $\Pi_{1}, \ldots, \Pi_{\mathrm{n}}$, where for any boolean condition $\Delta_{i}$ within an ifcond, $\Pi_{\mathrm{i}}$ represents either $\Delta_{i}$ or not $\Delta_{i}$.

An <activate id="norm"> activation parameters $</$ activate $>$ contract action specified within an ifcond is mapped to:

<initiates>

<event id="(pnorm, qualification)"> parameters </event>

$<\mathrm{fluent}$ id="norm" $>$ activation parameters

$</$ fluent $>$

condition

$</$ initiates $>$

Here an additional condition clause specifies that the contract action will only be applied if $\Pi_{1}, \ldots, \Pi_{\mathrm{n}}$ all hold.

Other atomic actions similarly have an additional condition clause when mapped.

Examples of such mappings for the Mail Service SLA are now presented.

- A violation event for $\sin 1$ initiates (or activates) pcmn3, and terminates (or deactivates) sln1_ok.

<initiates>

<event id="(sln1, violation)"/>

<fluent id="pcmn3"/>

$</$ initiates $>$

$<$ terminates $>$

<event id="( $\operatorname{sln} 1$, violation)" / >

<fluent id="sln1 ok"/>

$</$ terminates $>$

- A timeout event for pcnm1 initiates the assignment of (contract variable) vPenalty to 0 if the condition vPenalty greater than 0 holds.

<initiates>

<event id=" (pcnm1, timeout)" $/>$

$<$ mvfluent id="vPenalty">

$<$ num $\mathrm{val}=" 0 " />$

$</$ mvfluent $>$

$<$ gt $><$ value id="vPenalty" / >
$<$ num val="0" $/></$ gt $>$

$</$ initiates $>$

The mapping of the (possibly extant) contractual statement containing initialising operations for the contract (which is constrained to contain only activate actions) is now given (where norm is the norm activated). <activate id="norm"> activation parameters </activate> is mapped to:

<initially>

$<$ fluent id="norm" $>$ activation parameters

$</$ fluent $>$

$</$ initially $>$

Also, there is a mapping associated with the initialisation of contract variables in CTXML (where cvar is the contract variable assigned). <contractvar id="cvar"> expr </contractvar> is mapped to:

$<$ initially>

<mvfluent id="cvar" $>$ expr $</$ mvfluent $>$

$</$ initially $>$

For the full mapping to ecXML of the example SLA, see [16].

\section{Implementation}

The Event Calculus-based computational model has been comprehensively implemented in Java. The implementation provides a query-interpreter for determining, at run-time, the state of contracts, written in either ecXML or the higher-level CTXML. External components are able to post contract events via the queryinterpreter, and be informed of (and be able to query the contract for) information relating to contract state. Part of the API supported by this implementation is now presented.

- void get_output_events(ES,T)- gets, Es, the output events that the contract generates at time $\mathrm{T}$

- void get_states (S) - gets, s, the possible states of the contract

- void get_state_history $(H, T)$ - gets, H, a history of states that the contract has been in, up to and including time $\mathrm{T}$

- boolean active_at $(\mathrm{N}, \mathrm{T})$ - gives whether a norm, $\mathrm{N}$, holds at a time $\mathrm{T}$

- double value_at $(V, T)$ - gives the value of a contract variable, $\mathrm{V}$, at time $\mathrm{T}$

- void add_events(Es) - used to add an event narrative, Es, specified in ecXML, to the contract

- void add_future_events (Es, T) - used to add a future event narrative, Es, to the contract

- void delete_future_asserted_events() - used to remove all future events

Additionally, there is a means, provided for by the contract tracking ontology of defining equivalence classes 
for collections of contract states. It is the names of these equivalence classes that procedures such as get_state_history/2 return for names of states. An example of an equivalence class for the Mail Service UC SLA is: Customer Payment Outstanding, which describes all states for which there is an active obligation on the customer to pay.
A GUI called SLA Visualiser has also been implemented which manages the deployment lifecycle of UC SLAs. A snapshot of SLA Visualiser is shown in Figure 2. Here, SLA 4 is an instantiation of the Mail Service UC SLA presented in section 3. Figure 3 shows the history of SLA 4 in terms of the states it has been in, input and output events to and from the SLA and in other terms.

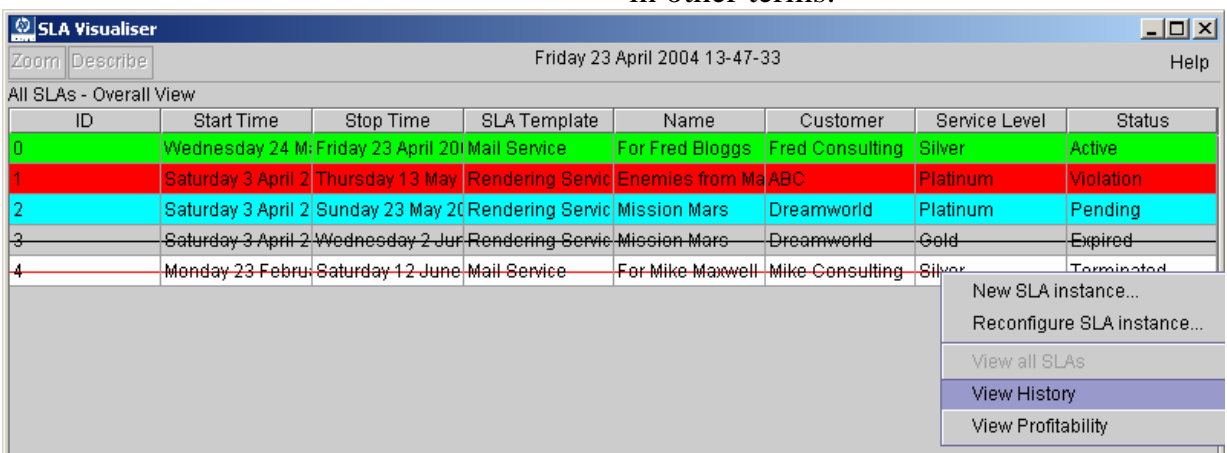

Figure 2: SLA Visualiser snapshot

\begin{tabular}{|c|c|}
\hline \multicolumn{2}{|l|}{ \$IA Visualiser } \\
\hline Friday 23 April 2004 13-48-25 & Help \\
\hline \multicolumn{2}{|l|}{ ustomer:Mike Consulting, Template:Mail } \\
\hline Occurrence & DaterTime \\
\hline STATE: OK & Monday 23 February 2004 13-47-20 \\
\hline STATE: Senvice Violation & Thursday 4 March 2004 13-47-20 \\
\hline INPUT EVENT: SERVICE VIOLATION with (slo: 1) & Thursday 4 March 2004 13-47-20 \\
\hline OUTPUT EVENT: OBLIGATION with (id: 0, bearer. provider, actions: resolve breach with (slo: 1), deadline: not specified) & Thursday 4 March 2004 13-47-20 \\
\hline STATE: OK & Thursday 4 March 2004 14-17-20 \\
\hline INPUT EVENT: SERVICE RESTORATION with (slo: 1) & Thursday 4 March 2004 14-17-20 \\
\hline INPUT EVENT: OBLIGATION with (id: 0 , status: fulfiled) & Thursday 4 March 2004 14-17-20 \\
\hline STATE: Provider Payment Outstanding, Customer Payment Outstanding & Friday 5 March 2004 13-47-20 \\
\hline \multicolumn{2}{|c|}{ OUTPUT EVENT: OBLIGATION with (id: 1, bearer: provider, actions: refund money with (amount: 25.00), deadline: before end of business day Friday 5 March 2004 13-47-20 } \\
\hline OUTPUT EVENT: OBLIGATION with (id: 2, bearer. Fred Consulting, actions: pay for service with (amount: 50.00), deadline: 1 month) & Friday 5 March 2004 13-47-20 \\
\hline STATE: Customer Payment Outstanding & Friday 5 March 2004 13-57-20 \\
\hline INPUT EVENT: OBLIGATION with (id: 1 , status: fulfiled) & Friday 5 March 2004 13-57-20 \\
\hline STATE: Terminable & Monday 5 April 2004 13-47-20 \\
\hline INPUT EVENT: OBLIGATION with (id: 2, status: timeout) & Monday 5 April 2004 13-47-20 \\
\hline STATE: Terminated & Tuesday 6 April 2004 13-47-20 \\
\hline INPUT EVENT: TERMINATE AGREEMENT & Tuesday 6 April 2004 13-47-20 \\
\hline
\end{tabular}

Figure 3: History of SLA

\section{Related work}

There have been many diverse research contributions that have utilised the Event Calculus (EC) for the purpose of reasoning over the effects of events on a logic theory. Those closest to the topics of this paper include $[3,4,8]$.

There has been a good deal of research concerning the representation of contracts for performance monitoring. In [6] Daskalopulu discusses the use of Petri-nets for contract monitoring, and assessing contract performance. Her approach is best suited for contracts which can naturally be expressed as protocols. One particular desirability of using Petri-nets is that they naturally facilitate analysis. In the context of contract representation, an example would be to show that a contract will always terminate in a favourable state for one, or more, contract parties. It is possible, however, to carry out analysis of this nature using the formalism described here. Moreover, our representation has many advantages over Petri-nets (some of which are as a result of a rule-based approach).

In [13] Milosevic and colleagues attempt to identify the scope for automated management of e-contracts; including: contract drafting, negotiation and monitoring. In [2], Abrahams defines the EDEE architecture (Ecommerce application Development and Execution Environment). Abrahams proposes Event-Condition Obligation rules for handling occurrences. Prima facie obligations are derived from the rules, where subsequent obligation choice decides which of these apply, and action choice decides which of those that apply will be fulfilled. In [9], Grosof and colleagues have sought to address the representation of business rules for e-commerce contracts. For this purpose, they have developed the SWEET 
(Semantic WEb Enabling Technology) toolkit, which enables communication of, and inference for, e-business rules written in RuleML. These approaches demonstrate many common themes with our approach.

\section{Conclusions}

In this work, we have proposed an ontology, formalised as an XML-based language, CTXML, to facilitate the automated tracking of contract state for performance monitoring. We have used the Event Calculus, defining a formalisation in XML called ecXML, to provide a computational model for CTXML. Through using EC, we are able to extract information regarding the state of contract norms - whether they are active or not - and variables - what value they have - for arbitrary times (in the past, or present), according to a supplied event narrative. It is also possible to simulate - using add_future_events $/ 2$ - the effects on contract state of a hypothetical event narrative, which we have found useful for carrying out prediction.

An inherent desirability of using EC is that the computation of tracking contract state - in the context of an event narrative - is externalised as a separate component, rather than buried within an implementation for contract monitoring. This promotes better modularisation and makes for simplified code maintenance. Also, as a consequence, it means that the state tracking component may be re-used for a range of automated reasoning tasks for which it is appropriate to track state.

A comprehensive Java-based implementation of a generic EC reasoning component, along with queryinterpreters for CTXML and ecXML, has been developed. $e c X M L$ can be seen as the 'language of the machine', and the implementation is capable of supporting any contract ontology that might be defined, so long as it has a tractable mapping to ecXML. All that is required to support a new ontology is the writing of a translator component for the ontology, which outputs ecXML. The ability to support multiple ontologies is an example of the re-use of the ecXML state tracking component.

The implementation and CTXML ontology have been evaluated against tens of SLAs, which are considered to be representative for UC. We have found the ontology to be sufficient for facilitating contract tracking (as defined in this paper) for these SLAs. We have also designed our implementation to be capable of supporting a high number of contracts simultaneously and to support event narratives with a very large number of events. We have optimised the implementation for querying, and have found it to work extremely efficiently. In the future, it is our intention to evaluate the sufficiency of CTXML at facilitating contract tracking for other sorts of SLAs, and for contracts from other domains.

The work described herein represents a small part of a larger effort considering a unifying approach to the management and utilisation of contracts, policies and business rules at all levels of a business enterprise, including: management of IT infrastructure and hardware, management of business processes using business rules authored by business managers and analysts, and management of agreements between trading partners. For more information concerning this work, see [16].

\section{References}

[1] Merriam-Webster On-line Dictionary (www.mw.com/cgi-bin/dictionary).

[2] A. S. Abrahams. Developing And Executing Electronic Commerce Applications with Occurrences. $\mathrm{PhD}$ thesis, Cambridge University, 2002

[3] A. Artikis. Executable Specification of Open NormGoverned Computational Systems. $\mathrm{PhD}$ thesis, Imperial College, London, U.K., 2003.

[4] A. K. Bandara, E. C. Lupu, and A. Russo. Using Event Calculus to Formalise Policy Specification and Analysis. In Proceedings of 4th IEEE Workshop on Policies for Distributed Systems and Networks (Policy 2003), Lake Como, Italy, June 2003.

[5] R. Boreham and M. Morciniec. Contract Monitoring. HP Labs Technical Report: HPL-2002-265.

[6] A. Daskalopulu. Modelling Legal Contracts as Processes. 11th International Conference and Workshop on Database and Expert Systems Applications, IEEE C. S. Press, pages 1074-1079, 2000.

[7] A. D. H. Farrell. Logic-based formalisms for the representation of Service Level Agreements for Utility Computing. Master's thesis, Imperial College, London, U.K., 2003.

[8] B. S. Firozabadi, M. Sergot, and O. Bandmann. Using Authority Certificates to Create Management Structures. In Proceedings of Security Protocols, 9th International Workshop, UK, April 2001.

[9] B. N. Grosof, Y. Labrou, and H. Y. Chan. A Declarative Approach to Business Rules in Contracts: Courteous Logic Programs in XML. In M. P. Wellman, editor, Proceedings of $1 s t$ ACM Conf. on Electronic Commerce (EC-99), Denver, CO, USA, November 1999. ACM Press, New York, NY, USA. 
[10] Hewlett-Packard (www.hp.com). HP Utility Data Centre - Technical White Paper. October 2001.

[11] R. Kowalski and M. Sergot. A Logic-Based Calculus of Events. New Generation Computing, 4:67-95, 1986.

[12] J. J. Lee and R. Ben-Natan. Integrating Service Level Agreements: Optimising Your OSS for SLA Delivery. Wiley, New York, 2002.

[13] O. Marjanovic and Z. Milosevic. Towards Formal Modelling of e-Contracts. In Fifth IEEE International Enterprise Distributed Object Computing Conference, Seattle, USA, September 2001.

[14] M. Salle and C. Bartolini. Management by Contract. HP Labs Technical Report: HPL-2003-186.

[15] M. Shanahan. Solving the Frame Problem: A Mathematical Investigation of the Common Sense Law of Inertia, ISBN: 0262193841. MIT Press, 1997.

[16] http://www.doc.ic.ac.uk/ adf02/phd 